Meta

Journal des tradlucteurs

Translators' Journal

\title{
De la pratique à la théorie, l'apport des practiciens à la théorie générale de la traduction
}

\section{Jean-Claude Gémar}

Volume 28, numéro 4, décembre 1983

URI : https://id.erudit.org/iderudit/002862ar

DOI : https://doi.org/10.7202/002862ar

Aller au sommaire du numéro

Éditeur(s)

Les Presses de l'Université de Montréal

ISSN

0026-0452 (imprimé)

1492-1421 (numérique)

Découvrir la revue

Citer cet article

Gémar, J.-C. (1983). De la pratique à la théorie, l'apport des practiciens à la

théorie générale de la traduction. Meta, 28(4), 323-333.

https://doi.org/10.7202/002862ar d'utilisation que vous pouvez consulter en ligne.

https://apropos.erudit.org/fr/usagers/politique-dutilisation/ 


\section{DE LA PRATIQUE A LA THÉORIE, L'APPORT DES PRATICIENS À LA THÉORIE GÉNÉRALE DE LA TRADUCTION*}

JeAn-Claude GÉmar

Depuis un quart de siècle environ on assiste à un phénomène qui ne laisse pas d'étonner le traducteur: l'activité qui occupe ceux qui, depuis quelque cinq mille ans ${ }^{1}$, se sont attachés à faire connaître, en s'essayant à les «translater» dans leur langue, les textes rédigés dans un idiome étranger, est de moins en moins le domaine réservé du traducteur, l'apanage du praticien. Des linguistes, en nombre croissant, se livrent avec un zèle parfois débordant à des actes de dissection de l'opération traduisante afin de l'analyser dans les moindres détails et de tenter d'expliquer de la manière la plus scientifique - qui correspond souvent à la plus théorique - possible le déroulement du processus traductionnel. Les traducteurs, relégués au second plan, en ressentent une frustration qui peut aller jusqu'à l'irritation lorsqu'ils croient reconnaître une tentative de détournement visant à faire de la traduction un sous-produit de la linguistique. Or l'action des uns n'exclut pas l'intervention des autres; je dirais même qu'elles sont dans une large mesure complémentaires comme nous allons voir tout à l'heure.

Depuis que l'homme traduit, il n'a cessé de s'interroger sur la nature de son art, sur la meilleure façon de faire passer d'une langue dans une autre l'intégralité du message contenu dans le texte de départ en s'efforçant d'en conserver le maximum de valeur sémantique et de dénaturer le moins possible ce qu'il est convenu d'appeler le "génie de la langue ${ }^{2}$ d'arrivée. Les grands traducteurs ont tous d'une manière ou d'une autre réfléchi sur l'opération traduisante et certains ont même exprimé sur la traduction des idées originales et profondes, mais aucun n'a vraiment exposé ses propos de façon systématique ni cherché à tirer de son expérience les éléments constitutifs d'un système,

* Ce texte est celui de la communication présentée par l'auteur aux SEDIFRALE 3 à Bogota en juillet 1981 .

1. Paul A. Horguelin (1981) : Anthologie de la manière de traduire. Domaine français, Montréal, Linguatech, p. 17, fait état de lexiques bilingues et même trilingues découverts sur le site de l'ancienne cité d'Ebla, dans le nord de la Syrie, datant du troisième millénaire avant J.-C.

2. Courante dans la terminologie canadienne (Vinay-Darbelnet, 1958), au sens de «Traits caractéristiques d'une langue» (Paul A. Horguelin (1978): Pratique de la révision, Montréal, Linguatech, p. 98), cette expression consacrée par la littérature (Stendhal, Montherlant...) et par la philologie (Littré), est critiquée par certains linguistes contemporains. 
les prémisses d'une philosophie de la traduction qui pussent servir de prolégomènes à une «théorie» de la manière de traduire, du moins au sens moderne de ce mot.

Il faut attendre la fin du XIX $x^{e}$ siècle et ses classifications, son souci de la hiérarchie linéaire et l'âge du positivisme pour voir s'amorcer une réflexion plus nourrie mais dont les racines, dans le trièdre épistémologique tracé par Foucault ${ }^{3}$, plongent encore aux sources de la réflexion philosophique traditionnelle plutôt qu'à celles des sciences empiriques ou même déductives qui, quelques décennies plus tard allaient pourtant servir de fondement aux théories «scientifiques» les plus hardies. Le vide épistémologique caractérisant une des activités les plus nobles et les plus anciennes de l'homme ne pouvait manquer d'attirer les futurs théoritrads et linguitrads ${ }^{4}$ dont les recherches sur les fonctions, le processus, le produit ou la didactique de la traduction ont conféré à une pratique traditionnellement considérée comme un «art littéraire» plutôt qu'une «science» le statut, envié et redouté à la fois, de discipline quasi scientifique.

Coincé entre les tenants des diverses théories linguistiques qui soit affirment qu'il est impossible de traduire, soit prétendent que les principales langues vernaculaires de la culture occidentale partagent un bagage minimal d'universaux suffisant pour rendre dans LA (la langue d'arrivée) l'essentiel du message porté par LD (la langue de départ), le traducteur est au coeur de la querelle doctrinale qui oppose depuis un quart de siècle environ les partisans des deux écoles. Plus que quiconque en effet, le praticien est en mesure de juger du bienfondé des arguments avancés par les deux parties puisque c'est à lui que revient la tâche, lourde et ingrate, de vérifier dans les faits, par le biais de l'expérimentation quotidienne, le cheminement de l'opération de transfert et d'établir le bilan de ses réussites et de ses échecs. Il est épaulé dans sa tâche par le pédago$\operatorname{trad}^{5}$, troisième et dernier intervenant dans le débat, qui constitue le ciment unissant la théorie à la pratique, la courroie de transmission indispensable sans laquelle toute communication entre théoriciens et praticiens serait lourdement hypothéquée.

La fonction du pédagotrad en fait le personnage clé autour duquel s'articulent désormais les lignes de force de la théorie générale de la traduction. Il sera intéressant de suivre l'évolution de ces trois groupes de personnages et de voir comment les praticiens, aux deux niveaux que représentent le traducteur et l'enseignant, pourraient finir par conjuguer leurs efforts pour jeter les bases d'une théorie générale et concrète de la traduction qui, tout en s'inspirant pour partie des découvertes de la linguistique générale, échapperait aux spéculations théoriques pures et trop souvent inapplicables des linguistes et intégrerait dans ses données les acquisitions de disciplines d'appoint ou complé-

3. Michel Foucault (1966): les mots et les choses, Paris, Gallimard, NRF, «Bibliothèque des sciences humaines», p. 355 et suiv.

4. J.-P. Vinay (1975) : «Regards sur l'évolution des théories de la traduction depuis vingt ans», Meta, XX, 1, p. 7-27, appelle ainsi les linguistes de profession qui cherchent à défricher le domaine de la traduction, selon le type de démarche adoptée. Cet article est assorti d'une importante bibliographie recensant les ouvrages théoriques majeurs.

5. Celui qui enseigne la traduction. $C f$. note 4 ci-dessus. 
mentaires telles que la sociologie, la philosophie, la psychologie, l'ethnologie, la communication, etc.

\section{LES ENSEIGNEMENTS DE L'HISTOIRE : 2000 ANS DE PRATIQUE}

Le traducteur, celui sur qui retombe «la série de problèmes pratiques qui (') assaillent journellement... ${ }^{6}$, est le premier artisan de son art comme en témoignent les très nombreux écrits que lui a inspirés l'opération traduisante. Le volume considérable de ce corps de doctrine et la grande quantité d'informations qu'il contient ont été peu exploités sur le plan théorique. On y retrouve pourtant en filigrane certains éléments des théories modernes de linguistique générale et, par voie de conséquence, des théories de la traduction, de l'antiquité romaine jusqu'à nos jours, exprimés selon le degré particulier de conviction et d'expérience de l'auteur et en fonction de son registre sociolinguistique : avocat, écrivain, philosophe, père de l'Église, érudit, poète, médecin ou homme d'État. Il n'est pas peu significatif de constater qu'un des pères fondateurs de la traduction, saint Jérôme lui-même, a le premier employé le mot «théorie» (du bas latin theoria, $\left.\mathrm{rv}^{\mathrm{e}} \mathrm{s}.\right)^{7}$ dans ses écrits, avec la fortune que l'on sait.

En introduisant dans le débat théorique sur la composition du signe linguistique le couple signifiant/signifié, Ferdinand de Saussure jetait les bases de la linguistique moderne mais également les fondements d'une théorie générale de la traduction toujours actuelle ${ }^{8}$. Par la suite, les théories (linguistiques) de la traduction ont fait intervenir plus particulièrement la notion de sens des mots, la sémantique, qui reste l'obstacle majeur en traduction. Si l'on admet que l'identification d'une difficulté ne garantit pas automatiquement qu'elle sera éliminée ou surmontée d'emblée, on peut néanmoins penser que l'on cherchera - et trouvera peut-être - les moyens de la contourner à l'avenir. Le cheminement du traducteur a suivi le parcours cahotique d'une course d'obstacles à mesure que se présentaient les difficultés particulières au genre dans lequel il exerçait son art: traduction de textes sacrés, littéraires ou scientifiques, selon la langue, l'époque et l'auteur.

Prenons pour exemple un grand traducteur et précurseur, Cicéron. Un choix fondamental est esquissé dans la manière de traduire qu'il expose dans son traité De optime genere oratorum :

...je ne les ai pas rendus en simple traducteur, mais en écrivain, respectant leurs

phrases avec les figures de mots ou de pensées, usant toutefois de termes adaptés à nos habitudes latines. Je n'ai donc pas jugé nécessaire d'y rendre chaque mot par un mot; pourtant, quant au génie de tous les mots et à leur valeur, je les ai conservés. (Je souligne.)

On l'aura compris, Cicéron prône ici le respect du signifiant qu'il privilégie par rapport au signifié. Mais pourquoi, au fait? Parce qu'il préconise l'imitation des auteurs grecs comme moyen de «se former à l'éloquence». De quoi s'agit-il, sinon d'une des fonctions (la fonction didactique) de la traduction. Ne nous

6. J.-P. Vinay, dans la préface du livre de Paul Horguelin, op. cit. note 1 ci-dessus.

7. Dictionnaire étymologique Larousse, Paris, Larousse, 1964, p. 744.

8. Comme en témoignent les Actes du Colloque international de Glendon (21-24 mai 1980), Meta, XXV, 4, et la plupart des communications, notamment celles de J. Darbelnet, D. Seleskovitch, Charles R. Taber, Louis Truffaut, J.-P. Vinay et Michael J. Gregory. 
hâtons donc pas de conclure que Cicéron était un tenant du littéralisme, de la forme opposée au fond. Prenons un autre exemple tiré cette fois de son traité De finibus bonorum et malorum:

Mais il ne sera pas toujours nécessaire de calquer votre langage sur le grec, comme le ferait un interprète maladroit [...] Pour moi, quand il s'agit de traduire, si je ne puis rendre avec la même brièveté ce qui ne demande aux Grecs qu'une seule expression, je l'exprime en plusieurs termes. (Je souligne)

Cicéron se contredirait-il? Il fait passer ici le signifié avant le signifiant. Que doit-on en conclure? Que Cicéron avait compris, sans qu'on le lui eût jamais enseigné, que la traduction est, à l'image de l'homme, au moins ambivalente ${ }^{9}$ : tantôt ange, tantôt bête aurait dit Pascal. Un autre (très) grand ancien évoqué plus haut, saint Jérôme, adopte également une attitude ambivalente dans ses traductions. Défendant avec ferveur dans son esquisse de traité de traduction, Lettre à Pammachius, De optimo genere interpretandi, sa conception de la manière de traduire, saint Jérôme déclare notamment :

Oui, quant à moi, non seulement je le confesse, mais je le professe sans gêne tout haut : quand je traduis les Grecs — sauf dans les Saintes Écritures, où l'ordre des mots est aussi un mystère - ce n'est pas un mot par un mot, mais une idée par une idée que j'exprime.

$\mathrm{Si}$, en théorie, saint Jérôme affirme sa volonté de traduire le sens et non les mots, ce qu'il a fait dans ses traductions du grec, en pratique la traduction de son cuvre majeure, la Vulgate, témoigne d'une fidélité surprenante au texte de la Bible. Ce conformisme s'explique par le souci de respecter la parole de Dieu : homme d'Église, saint Jérôme traducteur s'efface devant la divinité avec laquelle il ne prétend pas rivaliser. En revanche, lorsqu'il traduit les Pères de l'Église, il reconnaît: «Ce ne sont pas les mots, mais les idées que j'ai traduits. » Cet anti-conformisme déclaré viendrait, selon le professeur Truffaut, du fait que dans ces traductions «il rivalise avec des hommes qu'il peut égaler, sinon surpasser... ${ }^{10}$

On pourrait multiplier les exemples. Leur ancienneté n'en revêt que plus de valeur et de signification. Les premiers grands «translateurs» tel Jean de Meung, auteur de la seconde partie du Roman de la rose et archétype du traducteur littéraire, optèrent pour le signifié. Les premiers «traducteurs», à l'époque de la Renaissance, tels Étienne Dolet et Jacques Amyot, pour ne citer que les plus grands, optèrent eux aussi pour le signifié au détriment du signifiant, quitte à y laisser la vie comme dans le cas de Dolet qui fut brûlé vif pour avoir commis... deux faux sens révélateurs, fut-il jugé, de son athéisme et preuves d'hérésie. Finalement, ce choix entre deux ordres d'expression de la pensée ne revient-il pas à opter soit pour l'ordre établi - la traduction littérale étant la manifestation du respect de la lettre - soit pour l'évolution, le changement et l'imagination - la traduction du sens témoignant du respect de l'esprit. Choix fondamental, marque d'une grande indépendance d'esprit et de l'originalité de leur auteur. Que l'on pense à Cicéron, à Du Bellay, Rabelais, Luther ou

9. Voir sur ce point la remarquable communication de Louis Truffaut lors du Colloque de Glendon, «Les enjeux de l'ambivalence dans l'opération traduisante», Meta, XXV, 4, p. 430446.

10. Louis Truffaut, ibid., p. 431. 
Goethe, n'étaient-ils pas à leur époque des hommes libres, préfigurant un ordre nouveau?

Tout aussi probants sont la «vision du monde» et les aspects de civilisation dont témoignent les écrits de certains grands traducteurs tel Du Bellay, signataire du manifeste de la Pléiade et précurseur des théories néo-humboldtiennes lorsqu'il fait part de son scepticisme quant à la possibilité de traduire :

Je ne croirai jamais qu'on puisse bien apprendre tout cela des traducteurs, pource qu'il est impossible de le rendre avec la mesme grace dont l'auteur en a usé : d'autant que chaque langue a je ne scay quoi propre seulement à elle...

Deffence et illustration de la langue françoise (1549)

Plus édifiant encore est l'exemple de Martin Luther, précurseur de la traduction moderne comme «fait de société», placée devant de nouveaux problèmes linguistiques. Si Du Bellay ne fait qu'effleurer le problème de la relation entre signifiant et signifié ${ }^{11}$, $s$ 'approchant ainsi très près du problème du «sens», Luther fait lui aussi œuvre de précurseur en associant pour la première fois le texte à traduire au destinataire et en esquissant le principe de la conception synchronique de la traduction dans son Sendbrief vom Dolmetschen où il dépasse l'auteur du manifeste de la Pléiade :

Ce n'est pas aux mots de la langue latine qu'on doit demander comment parler allemand, c'est à la mère dans son foyer, aux enfants dans les rues, à l'homme du commun sur la place du marché qu'il faut le demander, en lisant sur les lèvres comment ils parlent, et c'est d'après cela qu'il faut traduire ${ }^{12}$. (Je souligne.)

Avec le siècle des «belles infidèles», le XVII ${ }^{\mathrm{e}}$, la tendance à adapter les textes que l'on traduit au goût et aux mours de l'époque s'accentue. Le destinataire est privilégié, le plus souvent au détriment de la vérité historique. D'après Roger Zuber, l'auteur de la célèbre étude sur Les belles infidèles et la formation du goût classique (1968), ces traducteurs n'ont pas peu contribué à l'élaboration du classicisme français. Le "siècle des lumières» verra se perpétuer cette tendance, non plus à l'encontre d'auteurs anciens, mais d'écrivains contemporains, les romanciers anglais en particulier. Rivarol, entre autres, dira qu'il faut traduire pour son temps, recréer «l'odeur du siècle».

Par un phénomène de «retour du pendule ${ }^{13}$, en réaction au libéralisme des siècles précédents, les traducteurs du XIX $x^{\mathfrak{e}}$ siècle reviennent au littéralisme érigé en système. Il faudra attendre la fin de la Deuxième guerre mondiale et l'explosion de l'activité traduisante qui s'ensuivit pour que les traducteurs, après un long silence, sous l'impulsion de fortes individualités comme Valery Larbaud et Edmond Cary, se prononcent en faveur du signifié contre le signifiant, pour la primauté de l'esprit sur la lettre. En même temps, la nature «littéraire» de la traduction, par opposition à son statut «scientifique", sera défendue avec éclat : pour Cary, la traduction est un art, non une science. Valery Larbaud estime, dans son essai placé Sous l'invocation de saint Jérôme, que le traducteur doit «éviter, d'une part le mot à mot insipide et infidèle à force de servile fidélité, et d'autre part la 'traduction ornée'». De son côté, Edmond Cary

11. Charles P. Bouton (1979) : la Signification, Paris, Klincksieck, p. 26.

12. Cité par Louis Truffaut, ibid., p. 432.

13. Paul A. Horguelin, Anthologie de la manière de traduire, op. cit., p. 147. 
affirme, dans son article «Défense et illustration de l'art de traduire», que «traduire c'est (...) autre chose que répéter ou copier.»

On me pardonnera ces longs exemples et l'arbitraire d'une sélection qui reflète mal mon dessein, ainsi que d'avoir recouru à de tels raccourcis historiques pour illustrer mon propos. La nature et le cadre de cet exposé limitent la portée de mes affirmations. Toutefois mon intention était de montrer que les premiers grands traducteurs, notamment jusqu'au $\mathrm{XVII}^{\mathrm{e}}$ siècle, avaient déjà identifié, le plus souvent de manière intuitive mais néanmoins saisissante, quelques-uns des principes fondamentaux des théories linguistiques de la traduction, contribuant ainsi à fonder la réflexion des théoriciens qui allaient trouver là un extraordinaire champ d'investigation, pratiquement vierge mais riche en virtualités. De l'œuvre des traducteurs se dégage une "philosophie de la traduction» qui s'inscrit dans la lignée des philosophes classiques et jette les bases théoriques d'une théorie générale de la traduction. Cet exceptionnel banc d'essai pour les spéculations théoriques de la linguistique interne devait devenir, comme l'histoire l'a démontré, la «chose» des linguistes, les traducteurs participant de moins en moins à l'élaboration d'une théorie générale et «scientifique» de la traduction.

La publication du Cours de linguistique générale (1916) de Ferdinand de Saussure marque une étape décisive dans l'histoire des théories de la traduction. C'est à partir des nouvelles orientations définies par ce grand linguiste que la théorie générale de la traduction devait se scinder en deux groupes irréductiblement opposés qui ont accentué le caractère manichéen du couple littéraire/scientifique, lettres/sciences, Anciens et Modernes, arbitrairement dissocié vers le milieu du XVIII ${ }^{\mathrm{e}}$ siècle ${ }^{14}$.

Dans la deuxième partie de mon exposé, je me propose de faire en premier lieu un rapide tour d'horizon des théories de la traduction en analysant, de manière très succincte et fort incomplète, l'apport des linguitrads et des théoritrads à la théorie générale de la traduction. Je ne m'étendrai pas sur cette question, amplement traitée dans de nombreux ouvrages, me réservant en second lieu de souligner la contribution extrêmement riche et positive d'une catégorie de praticiens qui n'appartiennent ni tout à fait au groupe des traducteurs professionnels - littéraires, scientifiques ou commerciaux, du monde des affaires ou du secteur public — ni entièrement à celui des théoriciens : je veux parler des pédagotrads cités plus haut. Mi-théoriciens mi-praticiens, les pédagotrads s'apparentent peut-être davantage aux didacticiens en ce sens qu'ils réfléchissent, à partir des conditions concrètes de leur expérience de traducteur, à la meilleure façon d'aborder les problèmes théoriques que pose la traduction et avancent des formules, des techniques et des méthodes pour les résoudre. Les critiques acerbes que suscitent chez les théoriciens ceux qui proposent des «recettes», jugées trop simplistes, au lieu de tenter d'élaborer des théories aussi savantes qu'inopérantes prouveraient, si besoin était, que c'est bien là que se situe le «scandale» dénoncé par Georges Mounin, qui est au centre

14. Il est intéressant de noter que Descartes ne faisait pas encore la distinction entre lettres et sciences lorsqu'il écrivit son Discours de la méthode (cf. Paris, Vrin, coll. Gilson, 1930, p. 4). 
du débat opposant les théoriciens qui formulent une objection «soit contre la légitimité, soit même contre la possibilité de toute traduction» ${ }^{15}$ et les praticiens qui «existent (et) produisent» et dont «on se sert utilement de (la) production $» 16$.

II. LA DIALECTIQUE TRADUCTIONNELLE OU LA RECHERCHE D'UNE SYNTHESE: PRATIQUE/THEORIE OU THÉORIE/PRATIQUE?

Qui ne connait la boutade de J.-P. Vinay déclarant «qu'il n'y a pas eu de théoriciens dans notre discipline pendant toute la période qui s'étend de Babel à $B a b e l »{ }^{17}$, soit jusque vers 1955 ? On peut avancer sans trop de risque qu'entre la théorie et la pratique, au début des années cinquante, la théorie de la traduction en était encore au stade du plus léger que l'air quand le traducteur en était déjà à celui de l'avion à réaction puisqu'il vole depuis près de 5000 ans de ses propres ailes. Il serait vain de croire cependant qu'aucune considération théorique n'est venue éclairer l'activité traduisante entre Ferdinand de Saussure, Fedorov, Vinay-Darbelnet et $\mathrm{Nida}^{18}$; au contraire, de nombreux linguistes avaient montré la voie aux futurs théoriciens de la traduction ${ }^{19}$. Il faut néanmoins reconnaître avec Georges Mounin que «la traduction, comme phénomène et comme problème distinct de langage, est passée sous silence ${ }^{20}$. Ce silence est désormais rompu et une avalanche d'ouvrages consacrés aux problèmes de la traduction, notamment linguistiques, est venue combler ce vide en quelques années au point de former un corps de doctrine considérable, tant en Europe qu'en Amérique du Nord. De ce flot il est possible de dégager trois courants principaux : (i) la traduction est théoriquement impossible; (ii) la traduction est une discipline accessoire de la linguistique; (iii) la traduction est la «discipline mère» de la linguistique.

Si l'on fonde l'étude scientifique ${ }^{21}$ du phénomène (deux langues en contact) que représente la traduction uniquement sur une analyse linguistique, il faut en conclure à la thèse de l'impossibilité de traduire parce que chaque langue organise la répartition de ses champs sémantiques de façon différente. À la limite, on ne pourrait donc faire coïncider un mot donné dans une langue avec son correspondant dans l'autre langue, comme l'a démontré Ferdinand de Saussure, par exemple, lorsqu'il compare le mot français «bœuf» avec son équivalent allemand Ochs. Le "sens" (Hjelmslev) étant construit de façon différente selon les langues, un «discours" (Guillaume) ne porte pas la même signification pour

15. Georges Mounin (1963): les Problèmes théoriques de la traduction, Paris, Gallimard, 290 p., rééd. 1976 dans la coll. TEL, p. 21.

16. Georges Mounin, ibid., p. 8.

17. J.-P. Vinay, Meta, XX, 1, op. cit., p. 8.

18. A.V. Fedorov (1953): Vvedenie v teorju perevoda (Introduction à la théorie de la traduction), Moscou; J.-P. Vinay et J. Darbelnet (1958), Stylistique comparée du français et de l'anglais, Paris, Didier; Eugene A. Nida (1964) : Toward a Science of Translating, Leiden, E.J. Brill.

19. Sur cette question voir plus particulièrement Georges Mounin, op. cit. chap. II.

20. Georges Mounin, ibid., p. 11.

21. Le signifiant scientifique est ici entendu au sens de "conforme aux exigences d'objectivité, de précision, de méthode des sciences» (Petit Robert), et non de simple méthode, quoique rigoureuse, d'analyse de certains mécanismes ou phénomènes. A cet égard, l'étude de la traduction par de nombreux chercheurs (essentiellement linguistes) contemporains peut être qualifiée de scientifique. 
toutes les catégories de lecteurs. De plus, chaque langue, selon les théories de Sapir et de Whorf (dites néo-humboldtiennes), édifie sa «vision particulière du monde", ou encore selon Lothar Kelkel "ouvre dans le monde ses propres chemins» quand elle n'établit pas une corrélation langage-culture (Claude Lévy-Strauss) particulière, en sorte qu'il faudrait un très grand hasard pour qu'elle coöncidât avec celle d'une autre langue.

A l'opposé, ou presque, Igor Mel'čuk voit dans la linguistique la «science de la traduction ${ }^{22}$. Partant du postulat que toute utilisation du langage est une traduction, Mel'čuk reprend et développe la thèse de Roman Jakobson pour qui "le sens d'un signe linguistique est sa traduction dans un autre signe" 23 . Autrement dit, "(t)out usage du langage humain n'est qu'un procès constant de traduction; la langue (au sens saussurien) est une machine traductrice... ${ }^{24}$. En conséquence, «il faut insister davantage sur les aspects «traductionnels» de la linguistique ${ }^{25}$.

Entre ces deux extrêmes, pour Vinay et Darbelnet la traduction est «une discipline auxiliaire de la linguistique ${ }^{26}$. Cette troisième option est la plus intéressante pour mon propos parce qu'elle représente l'un des aspects positifs du problème, celui où les implications méthodologiques sont les plus importantes, outre le fait qu'elle laisse entendre que la traduction, en tant que science «auxiliaire», peut être appelée à remplir certaines fonctions autres que linguistiques, comme nous le verrons.

Les limites des théories linguistiques de la traduction ressortent assez clairement lorsque du stade théorique l'on désire passer à celui de l'application, pour ne rien dire de la vérification. Posons la question: ces théories rendentelles plus facile la tâche du traducteur? Est-ce qu'elles lui tracent des règles, générales ou particulières, infaillibles qu'il peut suivre lorsque l'opération de transfert présente une difficulté inattendue? Force est de reconnaitre que «la linguistique qui revendique le statut de science exacte du langage n'a pas encore franchi le pas essentiel lui permettant de faire une unanimité sur ses présupposés propres ${ }^{27}$. La science a besoin de certitude, de durée; or une des caractéristiques de la traduction est son caractère fugitif: une traduction correspond à un moment précis de l'histoire linguistique d'un peuple et, en ce sens, la traduction est d'ordre synchronique et non diachronique, extemporanée même, pourrait-on dire, d'où la difficulté de lui conférer un statut scientifique permanent et de dégager des normes, de formuler des principes généraux applicables à toutes les langues que l'on désire traduire.

La Querelle des Anciens et des Modernes, qui renaît de ses cendres à intervalles réguliers ${ }^{28}$, en est la preuve depuis quelque 2000 ans en ce qu'elle

22. Igor Mel' $'$ ın, "Théorie de langage, théorie de traduction», Meta, XXIII, 4, p. 271-302.

23. Cité par Mel'čuk, ibid., p. 271.

24. Igor Mel'cuk, ibid., p. 271.

25. Ibid.

26. J.-P. Vinay (citant Vinay-Darbelnet, op. cit.), Meta, XX, 1, p. 15.

27. Charles P. Bouton, la Signification, op. cit., p. 68.

28. C'est l'éternel conflit entre la tradition et le modernisme, le conformisme et l'originalité. Elle a culminé au $\mathrm{XVI}^{\mathrm{e}}$ siècle (la Pléiade et son Manifeste) et surtout aux XVII ${ }^{\mathrm{e}}$ et XVIII ${ }^{\mathrm{e}}$ siècles, en France (Pascal, Boileau, Perrault...), en Angleterre (The Battle of the Books, Bentley, Temple, Swift...) et en Italie (Alessandro Tassoni). Au XIX ${ }^{\mathrm{e}}$ siècle, en Angleterre, la traduction servit 
oppose éternellement les partisans du signifiant - de la «norme» - aux tenants du signifié - l'originalité - et confirme si besoin était la théorie de la dérivation voulant que les mots, depuis leur origine, ne cessent de dériver $^{29}$. Les découvertes de la linguistique générale et théorique ont cependant permis aux traducteurs de prendre conscience de certaines difficultés que présente l'opération traduisante et d'établir nettement, par exemple, le rôle capital que joue le SENS (qu'il faut appréhender avant d'essayer de le rendre dans LA), c'est-à-dire le fond, par rapport au STYLE (la forme), sans aller jusqu'à affirmer comme le linguiste anglais J.R. Firth que «tout le problème de la traduction est d'ordre sémantique ${ }^{30}$. Sur ce point en tout cas traducteurs et linguistes sont d'accord: il faut traduire le sens, c'est-à-dire la signification moyenne d'un énoncé qui oscille plutôt entre une dizaine de possibilités réelles (dans un contexte donné) qu'entre les 28280000 paraphrases «possibles » calculées à partir d'un sens donné exprimable dans le cadre d'une phrase ${ }^{31}$. Or, tout comme dans le domaine de l'économie, la traduction se partage entre macro- et microscopie : le domaine de la macroscopie est celui du traducteur, le microscopique étant le fait du linguistique.

En résumé, quelle que soit l'option que l'on défend, on reconnaitra que la théorie générale de la traduction, notamment linguistique, est encore très insuffisante car elle n'explique pas vraiment le phénomène de la traduction et ne fait encore qu'effleurer le problème. En outre, nombreux sont les linguistes qui admettent que ce serait une erreur «que de vouloir enfermer la traduction, ses problèmes et ses solutions, dans les frontières de la linguistique ${ }^{33}$.

Cette dernière remarque nous amène à poser cette question : la traduction est-elle une discipline auxiliaire de la linguistique ou est-ce plutôt le contraire? Il est à l'heure actuelle banal d'affirmer que la traduction n'est pas une opération seulement linguistique, bien que cela reste toujours à démontrer. On sait maintenant avec certitude que la linguistique s'intéresse particulièrement aux fonctions du langage et que la traduction a pour principal objet la communication. À cet égard, celle-ci est le complément de celle-là.

Une forme de contribution particulièrement positive nous vient du troisième groupe évoqué tout à l'heure, celui des pédagotrads, qui s'est lui aussi

de prétexte à un nouvel épisode de la Querelle lorsque s'affrontèrent les partisans de Mathew Arnold et ceux de F.W. Newman à propos de la traduction d'Homère ( $c f$. On Translating Homer. Last Words, by Arnold). Plus près de nous encore, on peut citer la controverse que suscite la nouvelle traduction anglaise (par Terence Kilmartin) du chef-d'œuvre de Proust A la recherche du temps perdu (titre traduit par Remembrance of the things past, tiré d'un vers de Shakespeare, et conservé par Kilmartin), traduction que l'on oppose à celle de C. K. Scott Moncrieff, jugée inégalable. Proust s'était opposé, à l'époque, au titre proposé par Moncrieff. Certains critiques estiment que In Search of Lost Time serait une traduction plus exacte.

29. Michel Foucault, op. cit., p. 131.

30. Cité par Michael J. Gregory, in "Perspectives on Translation from the Firthian Tradition», Meta, XXV, 4, p. 455.

31. Voir sur ce point Igor Mel'cuk, "Théorie de langage, théorie de traduction», Meta, op. cit., p. 277.

32. Georges Mounin, op. cit., p. 227.

33. Eugene A. Nida, "Translating means communicating : a sociolinguistic theory of translation", in Linguistics and Anthropology (Muriel Saville-Troike, editor), Georgetown University Round Table on Languages and Linguistics 1977, Georgetown University Press, Washington, D.C., p. 213. 
étoffé au cours des vingt-cinq dernières années, et depuis la publication de la Stylistique comparée du français et de l'anglais (de Vinay et Darbelnet), a doté le monde de la traduction de la structure méthodologique de base nécessaire pour aborder de manière pratique mais formelle la plupart des problèmes que pose au traducteur l'opération de transfert. Les auteurs ont eu le mérite de comprendre la nécessité d'établir un répertoire des problèmes de traduction et de proposer une méthode pour les résoudre «en situation» et non dans le cadre de situations hypothétiques.

Une autre approche théorique mais inspirée des conditions pratiques de l'expérience complexe que représente la traduction de la Bible, est celle que nous propose Eugene A. Nida qui puise l'essentiel de sa réflexion dans la sociolinguistique. Bien que les nombreux exemples ponctuant systématiquement la démonstration représentent le plus souvent des cas extrêmes pour un traducteur - comme celui du missionnaire et des indiens de l'Amazonie pour qui le mot «bon» revêt un sens bien particulier... ${ }^{33}$ - ils n'en sont pas moins révélateurs d'une vérité, celle de la connaissance du «monde ethnographique»dans lequel évolue une langue et qu'il faut respecter, faute de quoi le traducteur aura fait ouvre incomplète ${ }^{34}$. C'est ici qu'apparaissent de la manière la plus crue les limites d'une théorie de la traduction reposant uniquement sur des considérations linguistiques. Un autre pédagotrad éminent, Danica Seleskovitch, ne parle pas d'autre chose lorsqu'elle dénonce les «doctrines» 35 - le mot théorie ne trouve pas grâce à ses yeux - linguistiques qui ignorent les «éléments cognitifs» du discours. Ces «compléments cognitifs» ${ }^{36}$ contribuent au sens de l'énoncé au même titre que le contenu sémantique des signes linguistiques.

Comme on voit, un nombre croissant de théoriciens nourris de pratique et de connaissances s'inspirant de disciplines tantôt extra-, tantôt paralinguistiques (sociologie, psychologie, ethnologie, théorie de la communication, philosophie...) sont en train d'édifier un corps de doctrine traductionnelle où la linguistique tient un rôle toujours essentiel, certes, mais non plus exclusif, de science auxiliaire de la traduction et non l'inverse. Les travaux récents de la nouvelle génération de chercheurs, entre autres ceux de Maurice Pergnier, J.-R. Ladmiral et Jean Delisle ${ }^{37}$, $s^{\prime}$ inscrivent dans cette perspective qui constitue, à mon sens, un des événements marquants de l'histoire de la théorie générale de la traduction des dernières années. Avec la prudence et l'humilité qui sont la marque des chercheurs qui s'aventurent en terrain mouvant, on

34. Georges Mounin épouse les vues de Nida, qui sont aussi les nôtres, lorsqu'il affirme : «Tout traducteur qui, de mille manières empiriques, ne s'est pas fait aussi l'ethnographe de la communauté dont il traduit la langue, est un traducteur incomplet. » (p. 239)

35. Le mot théorie, pour Danica Seleskovitch fait un peu présomptueux car il suppose que l'hypothèse a été vérifiée. $C f$. «Pour une théorie de la traduction inspirée de sa pratique», Meta, XXV, 4, p. 401-408.

36. Danica Seleskovitch, ibid., p. 406.

37. Maurice Pergnier (1978) : les Fondements sociolinguistiques de la traduction (thèse de doctorat), Paris, Champion, 491 p.; Jean-René Ladmiral (1979) : Traduire: théorèmes pour la traduction, Paris, Petite Bibliothèque Payot, $n^{\circ} 366$; Jean Delisle (1980) : l'Analyse du discours comme méthode de traduction, Ottawa, Éditions de l'Université d'Ottawa, coll. «Cahiers de traductologie», $n^{\circ} 2,1980,282$ pages. 
préfère parler des fondements, des théorèmes ou de l'analyse du phénomène complexe que représente la traduction, plutôt que de parler de «théorie».

\section{CONCLUSION}

Au terme de ces considérations, je voudrais tirer quelques rapides conclusions. Tout d'abord,

Si le travail de la science parvenue à maturité est justement de construire à partir d'un ensemble toujours limité d'observations et d'expériences, des hypothèses, des modèles théoriques, formulés de façon aussi explicite que possible et destinés à la fois à prévoir de nouveaux faits et à expliquer les anciens... ${ }^{38}$

la traduction est parvenue à ce stade de maturité après une longue gestation de deux mille ans. Ensuite, les efforts, enfin convergents depuis quelques années, des praticiens et des théoriciens ont au moins le mérite d'avoir fixé des limites au champ d'investigation des systèmes scientifiques théoriques et ouvert l'éventail des possibilités de recherche en intégrant dans l'analyse de l'opération traduisante les données les plus récentes de disciplines nouvelles ou traditionnelles, établissant ainsi le statut quasi scientifique et le caractère spécifique de la traduction, une des rares disciplines qui puisse se réclamer à la fois de l'art et de la science. N'est-ce pas, en fin de compte, le destin ambivalent de l'homme que d'être éternellement partagé entre le cœur et la raison? La traduction est le miroir où se reflète cette ambiguïté.

38. Charles P. Bouton, op. cit., p. 66. 